Die geopolitischen Interessen der transatlantischen Akteure USA und EU zur Ressourcensicherung sind heute zu konkurrierend und gegensätzlich, als dass sie unter dem Dach der NATO vereinigt werden könnten. In der Frage der militärischen, diplomatischen und ökonomischen Ressourcensicherung werden die transatlantischen Spannungen in den folgenden Jahren eher zu- als abnehmen, auch wenn die USA gegenwärtig an einer Stärkung und Aufwertung der NATO interessiert sind. Nicht auszuschließen ist allerdings, dass der Druck der russischen, chinesischen oder indischen Politik die USA und die EU sicherheitspolitisch wieder zusammenführen - allerdings kaum unter dem Dach der heute existierenden NATO.

\section{Alternativen jenseits westlicher Interventionen}

Wir müssen heute feststellen, dass Konflikte um Ressourcen auch im Kleid der Sicherung der Menschenrechte, Abrüstungskriege, des ,war on terror', der Militärpräsenz im Sinne der Sicherung einer ,good governance' oder künftig vielleicht im Gewand der ,responsibility to protect' auf uns zukommen.
Hermann Scheer betont den „weit reichendsten wirtschaftlichen Strukturwandel seit Beginn der industriellen Revolution“,45 der durch erneuerbare Energieträger ausgelöst werden kann. Auf den einleitend von ihm skizzierten „Energie-Imperialismus" kann heute eine weitere Fortschreibung der Besitzverhältnisse in Bezug auf alternative Energieformen befürchtet werden. Wenn Öl, Gas oder Uran nun durch Sonne, Wind und Wasser ersetzt werden, sich aber an den Besitzverhältnissen bei Kraftwerken, Leitsystemen oder der Distribution nichts ändert, wird die erwartete „Revolution“ ausbleiben. Eine demokratische und dezentral organisierte Substituierung der Energieträger ist nicht nur eine ökologische, sondern auch eine verteilungspolitische und friedenspolitische Frage. Die damit im Zusammenhang stehenden globalen Herausforderungen sind nichtmilitärischer Natur und verlangen nichtmilitärische und multilaterale Ansätze zu ihrer Bearbeitung. Weder die NATO Response Force noch die ,EU battle groups' können diese Probleme lösen.

45 Hermann Scheer, Energieautonomie. Eine neue Politik für erneuerbare Energien, München, S. 13.

\title{
Die geopolitische Dimension der Ressourcensicherheit - eine wachsende Herausforderung für Deutschland und Europa
}

\author{
Heinrich $\mathrm{Kreft}^{*}$
}

\begin{abstract}
Mineral raw materials are a key factor for sustainable growth in Germany and the EU. Unprecedented demand in particular from China has affected an increase in prices - a trend which is likely to continue. Germany and the EU are highly dependent on imports, including many high-tech minerals. Concerns that arise are thus the stability of producer countries, free access to raw materials and how to assist producer countries in managing resource extraction in a sustainable manner and to the benefit of their citizens. Resource security is a responsibility of private companies. But in order to address the growing political issues, an integrated approach is needed at the national, European and international level, which involves diplomacy, trade and development, as well as competitiveness, environmental and social policies.
\end{abstract}

Keywords: Rohstoffsicherheit, Importabhängigkeit, Hochtechnologiemetalle, Rohstoffdiplomatie, nachhaltiges Ressourcenmanagement

Security of resources, dependency on imports, high-tech metals, resource diplomacy, sustainable resource management

\section{Einleitung}

Rohstoffe sind für moderne Industrie- und Dienstleistungsgesellschaften unverzichtbar. Daher ist eine sichere Rohstoffversorgung zu akzeptablen Preisen von existenzieller Bedeutung für Deutschland und Europa.

\footnotetext{
* Dr. Heinrich Kreft ist Karrierediplomat mit Tätigkeiten an der Deutschen Botschaft in Bolivien, Japan und den USA. Zuletzt war er stellvertretender Leiter des Planungsstabs im Auswärtigen Amt. Derzeit ist er außen- und sicherheitspolitischer Berater der CDU/CSU-Bundestagsfraktion in Berlin.
}

Steigende Energiepreise und der wiederholte Gaskonflikt zwischen Russland und der Ukraine haben die Abhängigkeit Deutschlands und Europas von Öl- und Gasimporten für alle deutlich gemacht und auf die politische Agenda gesetzt. ${ }^{1}$ Vergleichbare Probleme bei der Versorgung mit nicht-energetischen Rohstoffen haben noch nicht diese Aufmerksamkeit

1 Zur Energiesicherheit Deutschlands und Europas, die hier nicht Gegenstand der Analyse sein kann, s. u.a. Heinrich Kreft, Die geopolitische Dimension der Energiesicherheit aus deutscher und europäischer Sicht, in: Reinhard C. Meier-Walser (Hrsg.), Energieversorgung als sicherheitspolitische Herausforderung, München, 2007, S. 31-50. 
der Politik erlangt, obwohl die Versorgungsrisiken, insbesondere bei einigen Hochtechnologiemetallen, höher sind als bei Öl und Gas.

Die Versorgungslage wird voraussichtlich auf Dauer angespannt bleiben, da aufgrund der Transformation zahlreicher Entwicklungsländer zu Schwellenländern die Nachfrage nach Rohstoffen auch zukünftig hoch bleiben oder sogar weiter zunehmen wird. Es besteht zudem die wachsende Gefahr von Rohstoffkonflikten in vielen Förderländern, angeheizt durch den international ungleichen Zugang zu diesen Rohstoffen sowie durch eine neue Handelsgeografie, hervorgerufen durch den Aufstieg der Schwellenländer.

Privatwirtschaft, Bundesregierung und EU-Kommission sind aufgefordert, durch geeignete Maßnahmen auf der Ebene der Unternehmen, des Staates bzw. der EU sowie auf internationaler Ebene nach Wegen zur Herstellung von Versorgungssicherheit zu suchen. Neben Maßnahmen zur Sicherung des Zugangs zu Rohstoffen, besteht ein wichtiger Weg zur Reduktion der Importabhängigkeit in der Steigerung der Ressourceneffizienz und in der Verbesserung des Recyclings von Alt- und Abfallstoffen.

\section{Fortbestehende Abhängigkeit von Rohstoff- importen}

Deutschland ist kein rohstoffarmes Land. Der Bedarf an Kiesen und Sanden, Steinen und Erden sowie Kali- und Steinsalz wird vollständig aus Rohstofflagerstätten in Deutschland gedeckt. Allerdings sind einige Rohstofflagerstätten - wie in anderen EUMitgliedstaaten auch - durch Schutzgebietsausweisungen und Festsetzungen mit anderen Nutzungen überplant und damit der Rohstoffgewinnung entzogen. ${ }^{2}$

Bei den meisten anderen Rohstoffen ist die deutsche Volkswirtschaft zumeist erheblich oder vollkommen von Importen abhängig, wie zum Beispiel bei Kupfer- und Eisenerz, die zusammen mit anderen zu importierenden metallischen Rohstoffen für eine Vielzahl von Branchen - von der Luft- und Raumfahrttechnik, dem Automobilbau bis hin zur Elektrotechnik und Elektronikindustrie - unverzichtbar sind. Insgesamt wurden 2007 Energierohstoffe, Metalle (Erze und Metalle der ersten Verarbeitungsstufe) und Nichtmetalle im Wert von 105 Mrd. Euro importiert.

Neben diesen Primärrohstoffen benötigt unsere Volkswirtschaft zudem in erheblichen Mengen sogenannte Sekundärrohstoffe, d.h. Schrott und Abfälle. Der Bedarf an Sekundärrohstoffen ist innerhalb der EU inzwischen auf 40 bis 60 Prozent des Ausgangsmaterials für die Metallerzeugung angestiegen. ${ }^{3}$

Ein großer Bedarfsträger von Rohstoffimporten ist die für unsere Volkswirtschaft so wichtige Exportindustrie. Rund 80 Prozent

2 Grundlage hierfür ist insbesondere der Grundwasser-, Natur- und Landschaftsschutz.

3 Schrott ist in der EU knapp geworden, da die Importe z.B. von Bunt- und Edelmetallschrott in die EU in den vergangenen acht Jahren um fast 40 Prozent zurückgegangen sind, während gleichzeitig der Export um 125 Prozent angestiegen ist. Ähnliches gilt für Eisenschrott. Ein besonderes Anliegen der Industrie ist daher ein wirksames Vorgehen gegen den illegalen Export von Sekundärrohstoffstoffen aus Europa auf europäischer Ebene. der Rohstoffe, die deutsche Unternehmen aus dem Ausland importieren, werden in veredelter Form wieder exportiert.

Eine hochgradige Importabhängigkeit besteht auch bei sogenannten Hochtechnologiemetallen wie Kobalt, Platin, Titan und seltenen Erden. Diese strategischen Rohstoffe sind für Schlüsseltechnologien und damit für die technologische $\mathrm{Zu}$ kunft unseres Landes unverzichtbar. Sie werden für die Herstellung technisch anspruchvoller Produkte, z. B. für die Umstellung auf eine nachhaltige Produktion und umweltfreundliche Produkte, zunehmend wichtig.

\section{Grundlegende Veränderungen auf den Welt- rohstoffmärkten}

\subsection{Verfügbarkeit von Rohstoffen}

Es ist zwar eine unumstößliche Tatsache, dass die Rohstoffausstattung der Erdkruste endlich ist, doch prinzipiell gibt es ausreichend mineralische Rohstoffressourcen, die den weltweiten Rohstoffbedarf auch langfristig decken können. Die seit der Jahrtausendwende zu beobachtende hohe Preisvolatilität auf den Rohstoffmärkten hat ihre Ursache nicht, wie oft irrtümlich angenommen, in der Erschöpfung von Rohstoffvorkommen. So beträgt die statistische Ressourcenreichweite von Chrom 600 Jahre und die der Platinmetalle noch immerhin 190 Jahre. Dennoch sind die Preise dieser und anderer Metalle mit mittleren Reichweiten in hohem Maße volatil. Die Marktturbulenzen sind das Ergebnis eines Ungleichgewichts zwischen Angebot und Nachfrage.

Die Rohstoffversorgung ist global hoch vernetzt und dadurch vielfältigen Einflüssen ausgesetzt, die von einem Staat oder gar einem einzelnen Unternehmen nur begrenzt beherrschbar sind. Marktstörungen können verschiedene Ursachen haben. Sie bestehen zumeist nicht in einer vollständigen Unterbrechung der Rohstoffzufuhr, sondern in Lieferverzögerungen, in Konzentrationsprozessen in der Bergbaubranche, mit negativen Folgen für den Wettbewerb oder in der Instabilität von Förderländern, was zum kurzfristigen Ausfall ganzer Produktionsstätten führen kann. Marktstörungen entstehen aber auch, wenn - wie in der Vergangenheit zu beobachten war - durch das rapide Wachstum von Entwicklungs- und Schwellenländern die Rohstoffnachfrage unvorhergesehen schnell ansteigt oder revolutionäre Technologieschübe Bedarfsspitzen oder auch Nachfrageeinbrüche zur Folge haben.

Das Störpotenzial ist dort besonders groß, wo keine (oder keine schnellen) Substitutionsmöglichkeiten für knappe und teure Rohstoffe existieren. So ist Chrom in rostfreien Stählen nicht ersetzbar, Kobalt nicht in verschleißfesten Legierungen, Indium nicht in Flüssigkristall-Displays und Flachbildschirmen, und Neodyn nicht in starken Permanentmagneten.

Zudem gibt es zwischen verschiedenen Wirtschaftssektoren Nachfragekonkurrenzen um bestimmte Rohstoffe. So wird Tantal in der Elektrotechnik, in der Stahlveredlung und in der Chirurgie benötigt, Platin in der Chemie und der Kfz-Industrie. 
Die starke Vernetzung der Rohstoffnutzungen in verschiedenen Industriesektoren ist bisher kaum erforscht worden. ${ }^{4}$

Die hohe Volatilität der Rohstoffpreise in den zurückliegenden Jahren geht zu einem großen Teil auf Fehleinschätzungen der Marktteilnehmer, insbesondere der Bergbauunternehmen, zurück. Eine erste Fehleinschätzung bestand darin, dass technologische Neuerungen nicht (rechtzeitig) erwartet wurden. Ein Beispiel dafür ist der starke Anstieg der Zinnnachfrage und des Zinnpreises als Folge des Umstiegs der Elektro- und Elektronikindustrie auf bleifreie Lote - denn diese benötigen einen höheren Zinnanteil. Die von neuen Technologien ausgehenden Impulse auf die Rohstoffnachfrage zeigt die gestaltende Kraft des technischen Wandels. Eine vom Institut für Zukunftsstudien und Technologiebewertung (IZT) zusammen mit dem Fraunhofer Institut für System- und Innovationsforschung (ISI) im Auftrag des Bundeswirtschaftsministeriums herausgegebene Studie ${ }^{5}$ geht der Frage nach, welche Impulse die zukünftige industrielle Nutzung von Zukunftstechnologien auf die Rohstoffnachfrage auslöst und auf welche Rohstoffe solche Innovationen besonders angewiesen sind. Bei einigen dieser Hochtechnologierohstoffe wird der Bedarf im Jahr 2030 auf ein Vielfaches der derzeitigen Weltproduktion geschätzt. So ist der von absehbaren technischen Innovationen ausgehende Bedarf an Gallium und Neodyn in 2030 sechs bzw. 3,8-mal so hoch, wie deren gesamte heutige Weltproduktionsmenge. Bedarfstreiber bei Gallium sind die Dünnschicht-Photovoltaik und schnelle integrierte Schaltungen, bei Neodyn die bereits genannten Hochleistungspermanentmagnete. Informationen darüber, welche Innovationen einen Nachfrageboom für bestimmte Rohstoffe auslösen, sind strategische Informationen für Rohstoffkonzerne, um ihre Produktionskapazitäten rechtzeitig an den zukünftigen Bedarf anpassen zu können.

Die zweite Fehleinschätzung betraf die stürmische Entwicklung der chinesischen Wirtschaft und den dadurch ausgelösten Importsog nach Rohstoffen, der für viele Marktteilnehmer unerwartet kam. Vor allem China hat dafür gesorgt, dass das jährliche Wachstum der Weltwirtschaft von über viele Jahre hinweg moderaten 3,8 Prozent seit 2004 auf fünf Prozent angestiegen ist. Angetrieben durch das hohe Wirtschaftswachstum Chinas erreichte der fünf Jahre anhaltende Rohstoff-Superzyklus Ausmaße, wie wir sie in der modernen Wirtschaftsgeschichte so noch nicht gesehen haben. Die globale Wirtschafts- und Finanzkrise hat die Preise für die meisten Metallrohstoffe zwischenzeitlich sinken lassen, allerdings auf ein für eine globale Wirtschaftskrise vergleichsweise hohes Niveau, von dem aus sie bereits wieder steigen.

Die Entwicklungen in China waren zwar der Hauptauslöser der jüngsten, von 2003 bis zur Wirtschafts- und Finanzkrise 2008/2009 herrschenden Rohstoffhausse, ${ }^{6}$ nicht aber ihre alleinige Ursache. Die Hausse ist auch auf das für die Rohstoffbran-

4 Institut für Zukunftsstudien und Technologiebewertung (IZT), Fraunhofer Institut für System- und Innovationsforschung (ISI), Rohstoffe für Zukunftstechnologien. Einfluss des branchenspezifischen Rohstoffbedarfs in rohstoffintensiven Zukunftstechnologien auf die zukünftige Rochstoffnachfrage, Schlussbericht - Kurzfassung, 2. Februar 2009, S. 12.

5 IZT/ISI, Rohstoffe für Zukunftstechnologien. Einfluss des branchenspezifischen Rohstoffbedarfs in rohstoffintensiven Zukunftstechnologien auf die zukünftige Rochstoffnachfrage, Schlussbericht, 15. Mai 2009.

6 Vom Anstieg der globalen Nachfrage nach Industriemetallen im Zeitraum 2002-2005 gingen über 50 Prozent allein auf das Konto Chinas. che typische zyklische Investitionsverhalten zurückzuführen. Indien spielt zwar derzeit noch keine große Rolle als Nachfrager auf den Rohstoffmärkten, dies dürfte jedoch angesichts der sich schnell entwickelnden indischen Volkswirtschaft nur eine Frage der Zeit sein. Nach der Bestätigung der reformorientierten Regierung von Premierminister Singh in den Parlamentswahlen vom Mai dieses Jahres ist davon auszugehen, dass Indien auf Reformkurs bleibt. China und Indien sind die einzigen größeren Volkswirtschaften, die laut Schätzung der Weltbank in der aktuellen Krise in diesem Jahr mit 8 bzw. 5 Prozent noch nennenswert wachsen werden.

Die hohen und selbst in der Krise (nach einem Verfall im vergangenen Jahr) gegenwärtig wieder ansteigenden Rohstoffpreise sind nicht das Ergebnis eines Angebotsschocks, sondern die Folge einer großen Nachfrage, die von einem starken weltwirtschaftlichen Wachstum ausgelöst wurde.

Für die Zukunft ist davon auszugehen, dass sich auch das Wachstum Indiens und anderer Schwellenländer merklich auf die Rohstoffnachfrage auswirken wird. Das wirtschaftliche Aufholen der weniger entwickelten Weltregionen - wie zum Beispiel Afrika - ist gewollt und Voraussetzung für eine friedliche Zukunft. Die Tatsache, dass die in der OECD zusammengeschlossenen Industrieländer bei nur 15 Prozent des Weltbevölkerungsanteils 50 Prozent $^{7}$ der mineralischen Ressourcen verbrauchen, ist sowohl aus politischen als auch moralischen Gründen auf Dauer nicht haltbar. Eine nachholende Entwicklung der armen Länder wird zwangsläufig das Weltwirtschaftswachstum über das langfristige Mittel der Vergangenheit steigen lassen. Aber selbst wenn das künftige jährliche Wirtschaftswachstum wieder auf Jahreswachstumsraten von 3,8 Prozent zurückfallen sollte, wird die Weltwirtschaftsleistung im Jahre 2030 bereits das 2,4-fache von 2006 erreichen, wovon zweifellos starke Impulse auf den zukünftigen Rohstoffbedarf ausgehen werden.

Die Nachfrage nach Massenrohstoffen, sogenannten Commodities, mit breitem Verwendungsspektrum wie Eisen und Stahl, Kupfer und Chrom, dürfte in der Zukunft stärker vom Weltwirtschaftswachstum getrieben werden, während die Nachfrage nach Hochtechnologiemetallen wie Gallium, Neodym, Indium u. a. m. eher vom technologischen Fortschritt bestimmt sein dürfte. Bei den Platinmetallen, Tantal, Silber, Titan und Kobalt dürften sich beide Treiber in gleicher Weise auf die Nachfrage auswirken.

Zu den Besonderheiten der Produktion metallischer Rohstoffe gehört ihr häufig gemeinsames („vergesellschaftetes“) Vorkommen in Erzen. Steigt die Erzproduktion aufgrund der größeren Nachfrage nach einem bestimmten Metall, werden auch andere damit gekoppelte Metalle in größeren Mengen produziert und umgekehrt. So wird z. B. das extrem knappe Indium als Koppelprodukt von Zink gewonnen. Als im Jahr 2006 eine japanische Zinkhütte geschlossen wurde, verringerte sich auch das Indiumangebot deutlich.

7 Beim Erdölverbrauch sind es sogar 56 und bei Erdgas etwa 60 Prozent. Selbst der durchschnittliche Rohstoffverbrauch Chinas ist pro Kopf um mehr als die Hälfte geringer als in den OECD-Staaten. 
Bei einigen Metallen wie Platin, Indium und Neodyn ist die Versorgungssicherheit aus drei gewichtigen Gründen als kritisch einzuschätzen. Zum einen handelt es sich dabei um strategische Rohstoffe für unsere Industrie. Zum zweiten ist nicht nur die Importabhängigkeit sehr hoch, sondern auch die Zahl der Lieferländer sehr klein. Drittens gibt es für diese Rohstoffe derzeit keine Substitutionsmöglichkeiten. ${ }^{8}$ China ist der größte Produzent von vielen Hochtechnologiemetallen, die es zunehmend in der eigenen im Aufbau befindlichen Hightech-Industrie einsetzt. Für etliche davon hat Peking bereits Exportbegrenzungen verfügt, was insbesondere da gravierend ist, wo China, wie bei Neodyn, mit 97 Prozent der Weltproduktion quasi über ein Weltmonopol verfügt.

\subsection{Neue Risiken durch gestörte Weltmärkte}

Die wichtigsten Fördergebiete für viele Hochtechnologiemetalle liegen in China, Afrika, Südamerika, Russland und Australien. Einige dieser Länder und Regionen sind wirtschaftlich oder politisch instabil, andere sind durch einen erheblichen Einfluss der Regierungen auf die Wirtschaft geprägt.

Schwellenländer verfolgen zunehmend Strategien, die darauf hinauslaufen, ihren eigenen, Rohstoffe verarbeitenden Industrien einen privilegierten und preisgünstigeren Zugang zu heimischen Rohstoffvorkommen zu sichern als ausländischen Konkurrenten. Mehr als 450 Exportbeschränkungen für mehr als 400 verschiedene Rohstoffe wurden von der EU festgestellt. ${ }^{9}$ Einige Schwellenländer zielen zudem darauf, sich privilegierten Zugang zu rohstoffreichen Ländern zu verschaffen. So hat sich China in den vergangenen Jahren an mehreren Großprojekten zur Erschließung von Rohstofflagerstätten in Afrika beteiligt. ${ }^{10}$

Typisch für eine Rohstoffpreis-Hausse ist die wachsende Konzentration und vertikale Integration der Unternehmen innerhalb des Bergbau- und Verarbeitungssektors. Bei anhaltender Entwicklung kann dies zu Einschränkungen des Wettbewerbs und damit zu höheren (Oligopol-)Preisen führen.

Über 50 Prozent der wichtigen Rohstoffvorkommen liegen in Ländern mit einem Pro-Kopf-Einkommen von unter zehn US Dollar pro Tag. Für viele dieser armen rohstoffexportierenden Staaten hat sich ihr Rohstoffreichtum als „Fluch“ erwiesen. Das Phänomen „Armut trotz Rohstoffreichtum“ lässt sich durch makroökonomische („Dutch Disease“) und politisch-institutionelle Defizite („Bad Governance“) erklären. Allerdings gibt es mit Chile und Botswana zwei löbliche Ausnahmen.

Mehr als die Hälfte der weltweiten Rohstoffproduktion erfolgt in Ländern, die von der Weltbank als politisch instabil oder extrem instabil eingestuft werden. Bei metallischen Rohstoffen stammen über 60 Prozent der Produktion allein aus instabilen oder extrem instabilen Ländern. Bei einigen Metallerzen

8 Im Jahr 2000 kam es beispielsweise infolge des Handy-Booms zu Versorgungsschwierigkeiten bei Tantal. Solche (temporären) Engpässe sind in Zukunft häufiger zu erwarten.

9 Zu den wichtigsten Ländern, die solche Maßnahmen ergriffen haben, gehören China, Russland, Ukraine, Argentinien, Südafrika und Indien.

10 U. a. in Sambia (Kupfer), DR Kongo (Kupfer, Kobalt), RSA (Eisenerz) und Zimbabwe (Platin). stammt sogar die gesamte Weltförderung aus Ländern dieser Kategorien. Das politische Risiko, dass diese Länder aufgrund von militärischen Konflikten, Terrorismus oder der Verstaatlichung von Rohstollquellen als Lieferanten ausfallen könnten, ist relativ hoch. Diese Gefahr besteht vor allem in Zentralafrika.

\section{Herstellung von Versorgungssicherheit in glo- balisierten Märkten}

\subsection{Materialeffizienz und Ressourcenproduktivi- tät erhöhen}

Die kritische Abhängigkeit der deutschen und europäischen Industrie von bestimmten Rohstoffen zeigt die Dringlichkeit eines Übergangs zu einer ressourceneffizienteren Volkswirtschaft und zu einer nachhaltigen Entwicklung. Dafür sollten Ressourceneffizienz, Recycling und die Substitution sowie der verstärkte Einsatz erneuerbarer Rohstoffe gefördert werden. Hierfür sind größere Investitionen insbesondere in leistungsfähige Recyclingtechniken notwendig, die kosteneffizient Sekundärrohstoffe in der Qualität von Primärrohstoffen gewinnen. Sekundärrohstoffe sind bei Metallen die einzige nennenswerte heimische Rohstoffquelle. Recycling verbessert zudem die Energieeffizienz, vor allem in der Metallerzeugung, da hier bei der Verarbeitung von Sekundärrohstoffen deutlich weniger Energie eingesetzt werden muss als bei der Verarbeitung von Primärrohstoffen.

\subsection{Erschließung heimischer Rohstofflager}

Um die Versorgung mit heimischen Rohstoffen sicherzustellen, muss die Erschließung neuer Rohstofflagerstätten ermöglicht werden. Dazu sollte die Bundesregierung im Zusammenwirken mit den Bundesländern eine gleichrangige Abwägung zwischen der Rohstoffgewinnung und anderen Belangen sicherstellen. Auch in der EU müssen die Rahmenbedingungen so gestaltet werden, dass eine dauerhafte Versorgung mit Rohstoffen aus europäischen Quellen begünstigt wird. Die dauerhafte Versorgung mit Rohsoffen aus heimischen Quellen setzt auch mehr Wissen über die in der EU vorhandenen Lagerstätten voraus. Durch eine stärkere Einbeziehung der staatlichen geologischen Anstalten, die sich zudem europaweit besser vernetzen sollten, in die Raumplanung könnte der Zugang zu Rohstofflagerstätten für eine spätere Erschließung offen gehalten werden. ${ }^{11}$

\footnotetext{
11 Kritik wird von der Bergbauindustrie vor allem an der Natura 2000-Richtlinie der EU geübt. Die Industrie fordert eine Anpassung, um sicherzustellen, dass in regelmäßigen Abständen eine Überprüfung der betreffenden Flächen unter gleichrangiger Abwägung der ökologischen, ökonomischen und sozialen Belange erfolgt, BDI, Intern, 10-Punkte-Papier zur Rohstoffpolitik vom 10.
} November 2008, S. 2. 


\subsection{Strategien zur Sicherung der Rohstoff- importe}

Rohstoffsicherheit ist ein volkswirtschaftliches Gut, für dessen Bereitstellung der Staat in enger Zusammenarbeit mit der Privatwirtschaft die Verantwortung trägt.

\section{Ebene der Unternehmen}

Die Rohstoffversorgung ist in erster Linie Aufgabe der Unternehmen selbst, aber nur weniger als die Hälfte der Firmen sichern sich derzeit gegen Risiken bei der Rohstoffversorgung ab. ${ }^{12}$ Die Unternehmen brauchen dafür einen eigenen strategischen Ansatz, der mit der Beschaffung strategischer Informationen über die Entwicklung des eigenen Bedarfs (unter Berücksichtigung des technologischen Fortschritts und Steigerung der Ressourceneffizienz), über drohende Rohstoffverknappungen und mögliche Preiserhöhungen beginnen muss. Den Firmen stehen verschiedene Instrumente unternehmerischer Rohstoffsicherung zur Verfügung, die auf die Sicherung des Rohstoffbezugs, auf die Erkennung und Abfederung von Rohstoffverknappung und -verteuerungen sowie auf die direkte Verständigung mit Rohstoffproduzenten und -lieferanten abzielen.

Eine Möglichkeit, den Rohstoffbezug zu sichern, ist die Beteiligung in der Rohstoffförderung („Rückwärtsintegration“). Hierdurch erhöhen sich die Chancen, den betreffenden Rohstoff in ausreichender Menge und Qualität zum benötigten Zeitpunkt zu beziehen. Durch eine solche Beteiligung lassen sich zu einem gewissen Grad auch die negativen Folgen von Anbieterkonzentrationen verhindern. Allerdings ist eine solche Beteiligung oder gar der direkte Kauf einer Rohstofflagerstätte mit hohem finanziellen Aufwand verbunden und damit für die meisten deutschen und europäischen rohstoffverarbeitenden Unternehmen keine Option.

Eine für die meisten Firmen realistische Option ist hingegen die Sicherung des Rohstoffbezugs durch langfristige Lieferverträge mit einer Bergbaugesellschaft, eventuell verbunden mit der Vereinbarung einer Partnerschaft. Daneben ist die Diversifizierung der Lieferbeziehungen ein klassisches Instrument der Risikostreuung. Auch durch Kooperationen im Einkauf, z. B. in Form gemeinsamer Einkaufsgesellschaften, können gerade kleinere Firmen ihre Nachfragemacht stärken. Zur Absicherung gegen plötzliche Rohstoffpreisanstiege existieren verschiedene finanzielle Absicherungsinstrumente (OTC-Forwards, -Swaps, Optionen und Bandbreitenstrategien). Solche Absicherungsinstrumente sind allerdings nur für solche Rohstoffe möglich, für die es einen offiziellen Referenzpreis (z. B. einen Börsenpreis) gibt. Ein weiteres Instrument ist die Pflege guter Geschäftsbeziehungen („Good Practice“) zwischen den Partnern einer Wertschöpfungskette. Hierdurch können existenzielle Krisen bei einzelnen Gliedern vermieden werden, bei gleichzeitiger Absicherung der Überlebensfähigkeit aller.

12 Hauck \& Aufhäuser, Risikomanagement von Rohstoffpreisen im deutschen Mittelstand, Unternehmerstudie 1/2009, Frankfurt.

\section{Staatliche Ebene}

Ein verlässlicher und von Marktverzerrungen unbeeinträchtigter Zugang zu Rohstoffen ist eine zunehmend wichtige Voraussetzung für die Wettbewerbsfähigkeit Deutschlands und der EU.

Aufgrund der hohen Abhängigkeit Deutschlands und der EU von Rohstoffimporten sind freie Weltmärkte ohne Handelsverzerrungen eine grundlegende Voraussetzung für die sichere Rohstoffversorgung. Allerdings sind die internationalen Rohstoffmärkte in beträchtlichem Maße von Handels- und Wettbewerbsverzerrungen gekennzeichnet. Insbesondere bedeutende Schwellenländer setzen in strategischer Weise handels- und wettbewerbsverzerrende Maßnahmen ein, um die eigene Rohstoffversorgung zu sichern. Infolge der Finanzmarktkrise kamen zusätzliche handelsverzerrende Maßnahmen zur Anwendung. Es ist Aufgabe des Staates, mit dem ganzen ihm zur Verfügung stehenden Instrumentarium für offene und funktionierende Weltrohstoffmärkte zu sorgen und für gleiche Wettbewerbsbedingungen für Unternehmen einzutreten.

Das politische Problembewusstsein ist in den vergangenen Jahren ohne Zweifel gewachsen: Die Bundesregierung macht Handels- und Wettbewerbsverzerrungen bei Rohstoffen inzwischen zum Gegenstand bilateraler Gespräche. Um diesem Problem wirksam begegnen zu können, hat die Wirtschaft den Vorschlag gemacht, die WTO-Regeln um ein Verbot von Exportbeschränkungen bei Rohstoffen zu ergänzen. Dies dürfte allerdings angesichts der divergierenden Interessen von Rohstoffexporteuren und -importeuren nicht einfach zu erreichen sein.

Auf bilateraler Ebene könnte erwogen werden, den Abbau von Exportbeschränkungen bei Rohstoffen und die Reduzierung wettbewerbsverzerrender Subventionen zur Bedingung für den Abschluss von Handelsabkommen und WTO-Beitrittsvereinbarungen zu machen. In bilateralen Gesprächen mit den betreffenden Ländern sollte konsequent auf den Abbau handels- und wettbewerbsverzerrender Maßnahmen hingewirkt werden.

Der staatlichen Ebene kommt zudem die überaus wichtige Rolle zu, mit ihrem außen- entwicklungs- und handelspolitischen Instrumentarium zur politischen und wirtschaftlichen Stabilität rohstoffreicher Länder beizutragen. Kurzum: Deutschland braucht eine aktive und integrierte Rohstoffdiplomatie.

\section{Ebene der EU}

Auch auf EU-Ebene ist eine aktive Rohstoffdiplomatie nötig. Die Europäische Kommission hat den Abbau von Handels- und Wettbewerbsverzerrungen bei Rohstoffen inzwischen zu einem festen Bestandteil ihrer bilateralen Verhandlungen mit den betreffenden Ländern gemacht.

Zur Sicherung der Versorgung Europas mit Rohstoffen wird ein ganzheitlicher Politikansatz auf europäischer Ebene angestrebt. Die politischen Herausforderungen für die Versorgung mit nicht-energetischen Rohstoffen sind vielschichtig und komplex; sie betreffen Wirtschafts- und Umweltpolitik ebenso wie Außen-, Handels- und Entwicklungspolitik. Die Verantwortung 
für viele der genannten Politikbereiche liegt heute ganz oder zum Teil bei der Europäischen Kommission. Die Entwicklung einer politikfeldübergreifenden EU-Rohstoffstrategie, die die nationalen Strategien sinnvoll ergänzt, ist daher erstrebenswert. Nach Aufforderung durch den EU-Rat für Wettbewerbsfähigkeit hat die Kommission inzwischen damit begonnen, einen solchen ganzheitlichen Politikansatz zu entwickeln. Sie hat im November 2008 einen Entwurf für eine Rohstoffstrategie vorgelegt, die auf drei Säulen basiert: Gewährleistung des diskriminierungsfreien Zugangs zu den auf dem Weltmarkt gehandelten Rohstoffen, Senkung des Primärrohstoffverbrauchs in der EU und Sicherung der Versorgung mit Rohstoffen aus heimischen Quellen. ${ }^{13}$ Zur Sicherung des Zugangs zu den benötigten Rohstoffen strebt sie ein besseres Management der bestehenden strategischen Partnerschaften ${ }^{14}$ sowie der vielfältigen Kontakte zu den meisten in diesem Kontext relevanten Staaten und regionalen Zusammenschlüssen an. Insbesondere mit Afrika soll der Dialog über den Zugang zu Rohstoffen im Rahmen des bestehenden Aktionsplans 2008-2010 intensiviert werden. Des Weiteren ist beabsichtigt, mit rohstoffreichen Schwellenländern wie Russland und China vor allem den Dialog mit dem Ziel einer Aufhebung marktverzerrender Maßnahmen (wie z. B. Exportbeschränkungen) zu intensivieren. Mit ähnlich rohstoffabhängigen Ländern wie Japan und den USA soll schließlich ein Dialog über gemeinsame Interessen und Initiativen zur Stärkung des freien Welthandels geführt werden. Außerdem will sich die EU für eine verstärkte internationale Zusammenarbeit engagieren und innerhalb der G8, OECD, UNCTAD und UNEP für ihre Position werben. Zu begrüßen ist auch das europäische Engagement in Zukunftsfragen - wie im Tiefseebergbau, in der Arktis und bei der Sicherung der internationalen Handelswege für Rohstoffe. ${ }^{15}$ Im Sinne eines ganzheitlichen Ansatzes setzt die EU auch auf ihre Entwicklungspolitik, um die staatlichen Strukturen in vielen schwachen Rohstoffförderländern zu stärken und eine nachhaltige Rohstoffbewirtschaftung zu unterstützen.

Daneben zielt die EU darauf ab, durch die Förderung eines günstigen Investitionsklimas zu einer Erhöhung des Rohstoffangebots beizutragen. Dazu gehört auch die Forderung an die Wettbewerbsbehörden der EU wie auch der Mitgliedstaaten, stärker auf die internationalen Konzentrationsprozesse in den Rohstoffsektoren zu achten, um die Versorgungssicherheit mit Rohstoffen zu angemessenen Preisen nicht durch Unternehmenszusammenschlüsse zu gefährden.

\section{Internationale Ebene}

Viele der für die Sicherung der Rohstoffversorgung Deutschlands und Europas als notwendig erachteten Maßnahmen sind weder bilateral noch auf EU-Ebene zu erreichen, sondern nur international und in Kooperation mit anderen interessierten Partnern umzusetzen. Dazu gehört eine bessere Kooperation

13 Mitteilungen der Europäischen Kommission, Die Rohstoffinitiative - Sicherung der Versorgung Europas mit den für Wachstum und Beschäftigung notwendigen Gütern, KOM(2008) 699, Brüssel, 4. November 2008.

14 Die EU hat strategische Partnerschaften mit China, USA, Russland, Indien, Japan, Brasilien und Kanada geschlossen.

15 Mitteilungen der Europäischen Kommission zu Rohstoffen, KOM(2008) 699, S. $6 \mathrm{f}$. in der Entwicklungszusammenarbeit wie auch internationale Transparenzinitiativen und andere Maßnahmen zur nachhaltigen Stabilisierung und Entwicklung rohstoffreicher Entwicklungsländer als Elemente eines globalen Ressourcenmanagements. In diesen Kontext gehören auch sogenannte nationale Rohstofffonds für rohstoffeiche Entwicklungsländer (z. B. in Zentralafrika). Wichtige Kriterien für den Erfolg solcher Fonds sind ein unabhängiges Management, auch um eine wirksame öffentliche Kontrolle zu ermöglichen, die Transparenz von Einnahmen und Ausgaben, um der Korruption entgegenzuwirken, sowie die Zweckbindung der Erträge für eine nachhaltige Entwicklung. Da viele Staaten zu schwach sind, um Rohstofffonds entlang dieser Kriterien aufzubauen und zu betreiben, sollten sie hierbei von internationalen Organisationen (z. B. von einer VN-Unterorganisation oder der Weltbank) unterstützt werden. ${ }^{16}$

Die Herstellung von Transparenz über Zahlungsflüsse ist ein wichtiges Mittel zur Korruptionsbekämpfung und für Good Governance. Die wichtigste Transparenzinitiative, die unter britischer G8-Präsidentschaft vereinbart wurde, ist die „Extractive Industries Transparency Initiative" (EITI). Die Initiative besteht in ihrem Kern darin, dass Zahlungsströme an öffentliche Stellen im Bereich der Rohstoffgewinnung, wie etwa Konzessionsabgaben oder Genehmigungskosten, von den Unternehmen offengelegt werden. EITI unterhält mittlerweile ein Sekretariat in Oslo und zahlreiche Staaten sind dieser Initiative inzwischen beigetreten. Die Initiative „Publish What You Pay“ verfolgt ähnliche Ziele, und die „Global Reporting Initiative“ zielt auf die Veröffentlichung von Umwelt- und Sozialdaten.

Der „International Council on Mining and Metals“ (ICMM) hat zehn Prinzipien für Nachhaltigkeitsstandards für seine Mitgliedsunternehmen entworfen - ein erster wichtiger Schritt zur Schaffung und Implementierung von Umwelt- und Sozialstandards. Neben dem seit Längerem bestehenden Kimberley-Zertifizierungsprozess ${ }^{17}$ für Diamanten ist von deutscher Seite eine Initiative zur Zertifizierung von Handelsketten im Rohstoffbereich ausgegangen. Die Bundesanstalt für Geowissenschaften und Rohstoffe hat ein Konzept erarbeitet, wie die Handelswege von Hightech- und Edelmetallen wie Tantal, Wolfram, Zinn und Gold speziell aus dem handwerklichen (artisanalen) Kleinbergbau überprüft und zertifiziert werden können. Mit Hilfe eines geochemischen „Fingerabdrucks“, der jedem Erz eigen ist, können illegal gewonnene und gehandelte Rohstoffe identifiziert werden. Gleichzeitig zeigt das Konzept, wie der Weg eines Rohstoffs auf der Grundlage internationaler Umwelt- und Sozialstandards vom lokalen Produzenten bis zu den industriellen Abnehmern zertifiziert werden kann.

Die Informationen über Rohstoffe und ihre Verwendung stammen aus ganz unterschiedlichen Quellen. Die Gründung einer internationalen Institution ${ }^{18}$ zur Beobachtung der Märkte könnte hier Abhilfe schaffen und den Bergbaukonzernen sowie

16 Vgl. Raimund Bleischwitz, Stefan Bringezu, Globales Ressourcenmanagement. Konfliktpotenziale und Grundzüge eines Global Governance-Systems, Stiftung Entwicklung und Frieden, Policy Paper 27, Oktober 2007 sowie den Beitrag von Bleischwitz in diesem Heft.

17 Nähere Informationen dazu unter: http://www.kimberleyprocess.com/.

18 Der United States Geographical Survey (USGS) ist die Institution, die derzeit die besten (aber nicht ausreichenden) öffentlich zugänglichen Rohstoffdaten und -analysen zur Verfügung stellt. 
den rohstoffnachfragenden Industrien aufbereitete Informationen zur Verfügung stellen. Informationsaustausch zwischen diesen Wirtschaftsakteuren dürfte zu einer besseren Abstimmung von Rohstoffangebot und -nachfrage führen und die risikobehaftete und damit kostenträchtige Volatilität auf den Rohstoffmärkten reduzieren.

\section{Ausblick}

Rohstoffsicherheit ist kein Selbstzweck, sondern entscheidende Voraussetzung für die Wettbewerbsfähigkeit der Industrie und damit für Wohlstand, Wachstum und Beschäftigung in Deutschland und Europa. Niemand kann die künftige Entwicklung der Rohstoffmärkte voraussagen. Die eingehende Analyse der treibenden Faktoren und die Herstellung von Transparenz über fundamentale Marktdaten erlauben es jedoch, belastbare Entwicklungstrends zu identifizieren. Dieser Trend geht in Richtung einer insgesamt steigenden Rohstoffnachfrage. Der Preisverfall im Zuge der globalen Finanz- und Wirtschaftskrise hat an der grundsätzlichen Bedeutung, unsere Rohstoffabhängigkeit zu reduzieren und sich um eine nachhaltige Sicherung unserer Rohstoffimporte zu bemühen, nichts geändert. Dadurch wird auch der schrittweise Aufbau eines Global-Governance-Systems für Ressourcenmanagement immer dringlicher, das am Eigeninteresse von Unternehmen und Staaten sowie an den aufgeführten internationalen Initiativen anknüpft. ${ }^{19}$

19 Bleischwitz und Bringezu schlagen hierzu drei Eckpfeiler vor: das „International Panel for Sustainable Resource Management“, ein Internationales Abkommen zum nachhaltigen Ressourcenmanagement und eine Internationale Agentur zum nachhaltigen Ressourcenmanagement, ebd. S. 9ff.

\title{
Präventive Ressourcensicherheitspolitik. Initiativen und Anforderungen an ein Global Governance-System
}

\author{
Raimund Bleischwitz*
}

\begin{abstract}
Resources of strategic importance are supposed to take centre stage in future conflicts. On this background, the author argues for the implementation of a preventive resource security policy in form of a multi-level approach that systematically includes non-governmental stakeholders. The main contribution of this article is the identification of some policy approaches and international initiatives relevant for a preventive resource security policy. In conclusion, the crucial importance of a global governance system is emphasized which includes an international agreement on sustainable resource management.
\end{abstract}

Keywords: Rohstoff- und Ressourcensicherheit, Transparenz, Ressourcenproduktivität, internationales Abkommen Security of raw materials and resources, transparency, resources productivity, international agreements

\section{Präventive Ressourcensicherheitspolitik - Rohstoffsicherheit plus Nachhaltigkeit}

Rohstoffsicherheit wird häufig auf den Zugang zu Rohstoffen und ihre kostengünstige Nutzung fokussiert. Eine Studie des US-amerikanischen National Intelligence Council (2008) entwirft ein beunruhigendes Szenario, wonach strategische Rohstoffe, die nur in wenigen Ländern abgebaut oder verarbeitet werden, im Mittelpunkt künftiger Konflikte stehen werden. Diese könnten sowohl bestehende Spannungen umfassen - genannt wird das Verhältnis zwischen China und Indien - als auch neue terroristische Attacken auf verwundbare Industriesysteme.

Derartige Konflikte könnten sich nicht nur bei Erdöl oder Erdgas ergeben. Die Rohstoffwirtschaft in Ländern Zentralafrikas und anderen schwachen Staaten bietet ideale Bedingungen für

Prof. Dr. Raimund Bleischwitz ist stellv. Forschungsgruppenleiter in der Forschungsgruppe Stoffströme und Ressourcenmanagement am Wuppertal Institut für Klima, Umwelt, Energie. schnelle Gewinne: Rohstoffe wie Cassiterit, Coltan, Gold oder Kobalt sind dort auf abenteuerliche Weise leicht zugänglich, erfordern wenig Transportaufwand und erzielen einen hohen Marktpreis. Dies ist nicht allein eine Einladung zu Schattenwirtschaft, illegalen Aktivitäten und lokalen Konflikten. Es kann sich auch eine Konfliktspirale ergeben, in die Nachbarstaaten, Unternehmen und westliche Industrieländer einbezogen sind.

Der folgende Beitrag argumentiert vor diesem Hintergrund für eine präventive Ressourcensicherheitspolitik. Darunter wird ein Mehrebenenansatz verstanden, der vorausschauende Strategien der Ressourcenschonung entwickelt und dabei gezielt nichtstaatliche Akteure aktiviert. Für eine solche Perspektive sprechen folgende Gründe:

Erstens geht es nicht um Rohstoffe als solche, sondern um ihre Funktion für menschliche Bedürfnisbefriedigung und Wohlstand. Die hohen Rohstoffpreise haben einen Paradigmenwechsel eingeleitet, in dessen Folge ein reines Angebotsdenken 